\title{
L'anti-romantisme ambigu de Drieu la Rochelle
}

\author{
Jean-Michel Wittmann
}

\section{(2) OpenEdition}

\section{Journals}

\section{Édition électronique}

URL : http://journals.openedition.org/studifrancesi/9744

DOI : 10.4000/studifrancesi.9744

ISSN : 2427-5856

\section{Éditeur}

Rosenberg \& Sellier

\section{Édition imprimée}

Date de publication : 1 octobre 2007

Pagination : 404-410

ISSN : 0039-2944

\section{Référence électronique}

Jean-Michel Wittmann, «L'anti-romantisme ambigu de Drieu la Rochelle », Studi Francesi [En ligne], 152 (LI | II) | 2007, mis en ligne le 30 novembre 2015, consulté le 09 janvier 2021. URL : http:// journals.openedition.org/studifrancesi/9744; DOI : https://doi.org/10.4000/studifrancesi.9744

\section{(c) (1) ()}

Studi Francesi è distribuita con Licenza Creative Commons Attribuzione - Non commerciale - Non opere derivate 4.0 Internazionale. 


\section{L'anti-romantisme ambigu de Drieu la Rochelle}

Engagé dans une polémique au sujet du symbolisme et de sa survie littéraire ${ }^{1}$, lui qui, dans État civil, venait d'évoquer «l'inévitable romantisme de [s]es dix-huit ans», présenté comme «une maladie que nous ne pouvons éviter, mais qui, dans l'état présent des mœurs, devient honteuse» ${ }^{2}$, Drieu la Rochelle est interpellé par Aragon. Dans sa «Lettre ouverte à Drieu la Rochelle» ${ }^{3}$, ce dernier observe que «romantisme est pris en mauvaise part». Après avoir ironiquement désigné ses contempteurs, «le sophiste Maurras et le Judas Moréas», il range sous une même bannière romantique «les plus grands: Bertrand, Nerval, Baudelaire, Rimbaud, Lautréamont, Cros, Nouveau, Apollinaire», et n'hésite pas à proclamer: «En face de toute convention, une seule force se lève, aux cris de la multitude. Oui, romantique» ${ }^{4}$.

Il faut dire qu'en ces années 1920, le terrain du débat autour du romantisme - combattu notamment par Maurras, qui lui oppose le classicisme - est bel et bien miné. Il s'agit non tant d'une question littéraire qu'idéologique et politique. Le feu allumé par Maurras à l'occasion du centenaire de la naissance de Hugo, entretenu notamment par la thèse de Pierre Lasserre sur le romantisme français, n'est pas éteint. Bien au contraire, en 1922, Léon Daudet, le pamphlétaire de l'Action française, vient de signer un pamphlet pour dénoncer Le stupide XIX siècle. Ce contexte donne son sens à la prise de position d'Aragon dans sa «Lettre ouverte à Drieu la Rochelle»; il permet aussi d'apprécier à sa juste valeur l'anti-romantisme singulier, voire ambigu, de ce dernier qui, à ce moment, s'apprête à rompre avec Aragon et les surréalistes, pour entamer une évolution qui le conduira au fascisme.

$\mathrm{Au}$ moment de considérer les jugements de Drieu sur le romantisme, on n'oubliera pas les influences personnelles dont il se réclame précisément. Dans État civil, il salue «la négation tranchante de Maurras ceignant étroitement son affirmation», après avoir évoqué «le nerf français tressé et durci par tous les excès, qui, dans le corps émacié de Barrès, se tendait encore après une âpre épreuve» ${ }^{5}$. Dans un article de 1920, il célébrait déjà «Barrès, Péguy, Maurras [qui] ont chanté la geste française du XIX ${ }^{\text {e }}$ siècle, l'aventure spirituelle d'un peuple» ${ }^{6}$. Il a lu passionnément le premier nommé, de son propre aveu: «Barrès est l'homme qui a eu sur moi le plus d'influence

(1) En réponse à un article de Jean Pierrefeu, paru dans le Journal des débats du 31 janvier 1923, qui évoquait le symbolisme en mentionnant son essai Mesure de la France, Pierre Drieu la Rochelle répond, dans Les Nouvelles littéraires du 10 février, en attirant l'attention sur «ceux qui reviennent de plus loin et qui sont encore engagés le plus bas dans le romantisme et le symbolisme». Aragon se prévaut de cette réponse pour interpeller à son tour Drieu: voir infra, et note 3.

(2) État civil [Gallimard, 1921], rééd. Gallimard, coll. «L'Imaginaire», 1986, p. 107.

(3) «Lettre ouverte à Drieu la Rochelle», repris in L. Aragon, Chroniques I, 1918-1932, Paris, Stock, 1998, pp. 137-138.

(4) «Lettre ouverte à Drieu la Rochelle», art. cité, p. 137.

(5) État civil, cit., p. 107.

(6) «Paul Adam», Nouvelle Revue Française, avril 1920, rééd. in P. DRIEU La Rochelle, Sur les écrivains, Paris, Gallimard, 1964, pp. 88-91. 
directe» ${ }^{7}$. Or Barrès, tout en prenant une part active à la croisade lancée contre le romantisme, au début du $\mathrm{XX}^{\mathrm{e}}$ siècle, n'a jamais cessé de se reconnaître lui-même comme un écrivain romantique. Il pouvait notamment accorder une place de choix à Rousseau dans son Panthéon personnel, tout en critiquant l'influence de Jean-Jacques dans un discours prononcé à la chambre des députés ${ }^{8}$; ou encore prendre acte du fait que son œuvre à lui se partageait à parts égales en «mode ionique» et «mode dorique»?.

L'ambiguïté de Drieu face au romantisme n'est pas moins remarquable que celle de Barrès. Dans ses romans comme dans ses articles ou ses essais, il n'a guère cessé de faire référence au romantisme. Implicitement ou explicitement, il doit se reconnaître comme l'héritier d'un mouvement qui domine le XIX siècle au point de se confondre avec lui. Pourtant, s'il exerce un droit d'inventaire face à cet héritage, il ne manque pas d'en souligner la grandeur. Il affirme par exemple: «le romantisme, c'est toute l'Europe pendant une de ses plus belles saisons», à l'occasion d'un hommage rendu à Mickiewicz, «grand poète romantique» qui appartient, comme tel, à «cette race de géants qui a foisonné pendant cent ans et qui a fait du XIX ${ }^{\mathrm{e}}$ siècle une époque aussi majestueuse que celle de la Renaissance» ${ }^{10}$. Convaincu d'avoir été confronté, comme toute sa génération, à «un fait écrasant: la décadence» ${ }^{11}$, il présente tour à tour le romantisme comme le symptôme d'une renaissance ou, au contraire, d'un processus de décadence. Il peut ainsi sembler opposer un romantisme plein de vie, celui du XIX siècle, à celui du temps présent, où il subsiste à l'état de «maladie honteuse», pour reprendre le terme d'État civil; mais en réalité, il perçoit ce mouvement même comme essentiellement ambivalent, dès ses origines.

C'est assurément dans ses Notes pour comprendre le siècle qu'il expose le plus clairement cette ambiguité, à tel point que cet essai de 1941 éclaire rétrospectivement les références au romantisme dans son œuvre et ses jugements sur le mouvement, qui pourraient sembler dépourvus de cohérence. On n'aura garde d'oublier que ce livre a été écrit dans un contexte particulier, à un moment où il s'agit pour Drieu de justifier ses positions politiques, son attirance pour le totalitarisme et, en particulier, les espoirs qu'il a placés - un temps - dans l'Allemagne nazie. Pourtant, cet essai tire précisément sa force et son intérêt du fait que Drieu y mène en parallèle une réflexion générale sur l'histoire et des développements sur l'évolution de la littérature française.

Dans ces Notes pour comprendre le siècle, les vues développées par Drieu reposent sur le postulat suivant lequel la santé de l'homme réclame l'équilibre entre le corps et l'esprit, atteint lors des périodes florissantes, dans l'histoire de la civilisation. Dans cette perspective, il semble à Drieu qu'au Moyen Âge, époque virile par excellence, a succédé une période de progressive dégradation de cet équilibre, à mesure que triomphait le rationalisme. Le XIX ${ }^{\mathrm{e}}$ siècle, tout particulièrement à sa fin, marque une réaction contre ce rationalisme et tend vers un rétablissement de cet équilibre jugé primordial par Drieu, d'une part, parce que l'homme désormais enfermé dans des villes a commencé alors à cultiver son corps par le sport, et d'autre part, parce que

(7) Drieu la Rochelle à Frédéric Lefèvre, en 1926, propos cités par F. Grover in Sur les écrivains, cit., p. 247.

(8) Voir notre essai, Barrès romancier. Une nosographie de la décadence, Paris, Champion, 2000, p. 38 .

(9) Voir Mes Cabiers, in L'ouvre de Maurice Barrès, t. XIV, Paris, Club de l'honnête homme, 1968, p. 90.
(10) Préface à M. Czapskar, La Vie de Mickiewicz, Paris: Plon, 1931, p. 13.

(11) Voir sa préface à Gilles [Gallimard, 1942], rééd. Gallimard, coll. «Folio» n 459, 1992, p. 10; Drieu y analyse le développement de son œuvre en partant du constat suivant: «Je me suis trouvé comme tous les autres écrivains devant un fait écrasant: la décadence». 
le romantisme et, plus encore, le symbolisme, traduisent un nouvel élan spirituel. Dès lors, l'essayiste peut affirmer: «Toute cette histoire du romantisme et du symbolisme [...] c'est tout simplement l'histoire de la mysticité française renaissante, de l'esprit français s'arrachant au rationalisme» ${ }^{12}$...

Le jugement de Drieu sur le romantisme et le symbolisme est cependant plus complexe que ne le laisse supposer cette thèse, ainsi résumée. Pour lui, le romantique est «un homme qui a douté de la raison en voyant le spectacle de 1789 à 1815», mais qui «s'y raccroche désespérément»: «il voit les inconvénients du rationalisme, la rupture entre raison et mystique, mais il ne peut refaire le nœud $\gg^{13}$. Cet échec trouve à la fois un écho et une détermination dans les soubresauts révolutionnaires du XIX ${ }^{\mathrm{e}}$ siècle, condamnés par Drieu: «Tous commencent par l'inquiétude, l'effroi, le gémissement devant les ruines de l'histoire. [...] Mais tous, tant bien que mal, renouvellent l'effort et le défi rationalistes en 1830, en 1848; et, après l'échec de 1848, le naufrage de 52, le naufrage de 70 et 71 , ceux qui restent le renouvellent encore sous une forme définitivement abâtardie dans la IIIe République» ${ }^{14}$.

Certes, c'est le symbolisme qui, dans la perspective de Drieu, manifeste véritablement une réaction contre le rationalisme. Comme l'observe justement Frédéric Grover, pour Drieu, «le romantisme, bien qu'il semble réagir contre ce rationalisme, est contaminé par lui», mais «la littérature française se redresse dans le magnifique élan du Symbolisme (pris dans un sens très large) $\rangle^{15}$. Très large, effectivement, puisqu'il englobe pratiquement le naturalisme et la littérature de la décadence, le symbolisme, tel qu'il est présenté dans Notes pour comprendre le siècle, reproduit pourtant, et même accentue, les ambiguïtés essentielles du romantisme. Aussi bien, dans Genève ou Moscou, après avoir présenté le romantisme comme un «va-et-vient industrieux sur toutes nos frontières mentales», Drieu évoque le moment où «Barrès, Maurras, Péguy, Claudel et Gide» entraient en littérature comme celui où «tout cela continuait de fermenter dans ce magnifique atelier d'essai et de transformation qu'a été le symbolisme» ${ }^{16}$.

Il n'est que de considérer le jugement porté sur Mallarmé dans les Notes pour se convaincre de l'ambiguité de Drieu à l'égard du symbolisme, qu'il définit au demeurant comme «le romantisme vrai» ${ }^{17}$. «Il y a chez Mallarmé le dernier degré de la déchéance du corps, la déchéance sexuelle», observe Drieu, qui poursuit en présentant la poésie du chef de file du symbolisme comme «le chef-d'œuvre de l'onanisme». Et l'essayiste de conclure à son sujet: «Autour de ce point délicat se résout définitivement la défaillance du siècle», avant de brosser le portrait de cette génération, qui lui apparaît marquée par la décadence: «Après l'Anatole France des premiers romans si désolés, le Barrès de Sous l'œeil des Barbares, Proust décrira ces gnomes de la bourgeoisie finissante. Après Huysmans, voici Louÿs, Jean Lorrain, qui se chargent de la nomenclature détaillée du vice. Gide établit dans Les Caves du Vatican la dissolution de la morale, la rupture définitive de l'individu intellectuel avec la société» ${ }^{18}$.

De ce point de vue, le symbolisme, qui a «poussé à fond [...] une des deux tendances du romantisme, [...] la mélancolie, le chagrin, le désespoir de l'âme destituée, séparée du corps, retournée sur elle-même» ${ }^{19}$, ce n'est que le romantisme en pire! Drieu n'a-t-il pas auparavant présenté «l'homme romantique», «talonné par le

(12) Notes pour comprendre le siècle, Paris, Gallimard, 1941, p. 118.

(13) Ibid., p. 70

(14) Ibid., pp. 70-71.

(15) F. GROver, Drieu la Rochelle, coll. «La Bibliothèque idéale», 1962, pp. 162-63.
(16) Genève ou Moscou, repris in Sur les écrivains, cit., p. 134.

(17) Voir Notes pour comprendre le siècle, cit., p. 110 .

(18) Ibid., pp. 100-102.

(19) Ibid., p. 99. 
chagrin de ne pouvoir être que rationaliste et de ne pouvoir redevenir solidement mystique», comme un «homme fatigué, [dont] il faut bien reconnaître qu'il a encore en lui de grandes ressources d'énergie», avant d'ajouter: «Il essaie aussi de s'en tirer, par un formidable mouvement de science, d'industrie, de voyage. Balzac se passionne pour la science et l'industrie; de même Hugo, Lamartine, Vigny» ${ }^{20}$ ?

De l'essor du romantisme jusqu'à l'avènement du symbolisme et à son déclin au début du $\mathrm{XX}^{\mathrm{e}}$ siècle, l'histoire de la littérature coïnciderait donc avec un mouvement de dégradation continue, mais ce constat pousse Drieu à tirer une conclusion paradoxale: «Dans le pire, l'homme touche terre et rebondit. Le corps et l'âme arrivés au fond de la dégradation renaissent en même temps. Dans ce symbolisme qui renferme toutes les corruptions, j'aperçois le germe d'une renaissance de l'âme» ${ }^{21}$. D'une génération à l'autre, plus on se donne les moyens de lutter contre la maladie - la décadence -, et plus on est malade! On comprend donc pourquoi Drieu, dans État civil, condamne «l'inévitable romantisme de sa jeunesse» ${ }^{22}$, assimilable à une force dégradée, usée par les générations successives. Pour accélérer le processus de décadence, le romantisme, comme le symbolisme plus tard, n'en apparaît pas moins comme un effort pour réagir contre les facteurs de cette décadence. Aussi l'homme contemporain semble-t-il condamné à assumer l'héritage romantique, pour le pire, mais aussi pour le meilleur: voilà la source de l'ambiguïté propre aux références et aux allusions au romantisme dans l'œuvre romanesque de Drieu.

Présenté comme une force dissolvante, moralement et socialement, le romantisme y fait généralement l'objet d'une critique sans équivoque. Ainsi, dans Le Feu follet, où la maison de santé tenue par le docteur de la Barbinais devient la métaphore d'une société décadente, peuplée d'individus malades et affaiblis. Ces derniers, qui reflètent la «déchéance de l'homme ${ }^{23}$ évoquée dans Notes pour comprendre le siècle, se regardent dans le miroir tendu par les écrivains romantiques, dont ils sont les enfants, pour ne pas dire les victimes: «Le docteur de la Barbinais n'avait pas craint d'aligner sous les yeux des neurasthéniques qu'il soignait le portrait de tous les écrivains qui depuis deux siècles s'étaient rendus célèbres par leurs chagrins» ${ }^{24}$. Alain lui-même, le personnage principal, est présenté comme «un être dont toutes les lâchetés devant la rudesse de la vie s'étaient conjurées depuis longtemps pour le maintenir dans cette dérobade complète du paradis artificiel» ${ }^{25}$. La neurasthénie d'Alain, comme celle des autres pensionnaires de cette maison de santé, c'est «la sentimentalité mélancolique qui passe aussi à Hugo, et se prolonge en morosité jusqu'à la fin du XIX siècle, à travers Flaubert, le premier Zola, Leconte de Lisle, Mallarmé et Barrès ${ }^{26}$. Encore Alain, capable de se suicider, est-il l'épigone d'un Nerval ou d'un Baudelaire, chez qui cette sentimentalité, «en se poussant au dernier point du désespoir, [...] commence de le surmonter, elle rejoint et dépasse Pascal, elle tend à Nietzsche» ${ }^{27}$.

S'il critique ainsi une forme de sensibilité qui coupe du réel et bride l'action, Drieu apparaît en revanche nuancé dans son approche de l'égotisme. Dans ses Notes pour comprendre le siècle, il ne laisse pas d'être admiratif face à Stendhal, le promoteur de cet égotisme moderne. Stendhal lui apparaît «certes touché par le mal du siècle, mais il contient ce mal par la tendance à l'action, le mouvement du voyage et de la curiosité, le goût du plaisir fin et des idées claires et praticables ${ }^{28}$. Savoir jouir

(20) Ibid., pp. 71-72.

(21) Ibid., pp. 105-106.

(22) Voir supra, et note 2.

(23) Voir Notes pour comprendre le siècle, cit., p. 91: «Quelle déchéance de l'homme tels que les romanciers le décrivent, de Stendhal à Zola!»
(24) Le Feu follet [Gallimard, 1931], rééd. Gallimard, coll. «Folio» $\mathrm{n}^{\circ} 152,1986$, p. 23.

(25) Ibid., p. 46.

(26) Notes pour comprendre le siècle, cit., p. 68.

(27) Ibid.

(28) Ibid., p. 69. 
des ressources de sa sensibilité, c'est ce qui caractérise encore Stendhal, même si cette sensibilité peut prendre un tour pathologique chez ses personnages: «Il y a évidemment de la neurasthénie dans la tension concertée de Julien Sorel, de Fabrice, de Lucien Leuwen, de Lamiel; mais par en dessous, il reste beaucoup d'aisance à vivre, à agir, à penser, à jouir» ${ }^{29}$.

Il est entendu pour Drieu que la neurasthénie semble devoir prendre le pas sur l'aisance à vivre chez les héritiers contemporains de Stendhal, comme en témoignent les personnages de Rêveuse bourgeoisie, réécriture fictionnelle de son «roman familial». Geneviève lit «éperdument Le Rouge et le Noir» dont son frère Yves lui a conseillé la lecture, juge «pressé» de connaître Mathilde de la Môle, mais tous deux, et tout particulièrement Yves, l'initiateur à Stendhal, avec «son petit corps délicat, ultrasensible d'enfant solitaire vivant près des femmes» ${ }^{30}$, sont présentés comme des êtres essentiellement faibles, velléitaires, incapables de transformer en véritable force les ressources de leur sensibilité.

L'égotisme, pour être tourné à bien - processus qui reviendrait à tirer bénéfice du romantisme, en rejetant le germe négatif qu'il comporte -, doit être corrigé suivant les principes du Culte du Moi barrésien. Dans Un Homme libre, livre de chevet de Drieu, Barrès définit un programme qui fixe les objectifs d'une culture du moi bien comprise: «sentir le plus possible en analysant le plus possible»; mais il conduit son héros à comprendre que «les individus [...] ne sont que des fragments du système plus complet qu'est la race, fragment elle-même de Dieu» ${ }^{31}$. De même, le héros d'Une Femme à sa fenêtre, Boutros, est présenté comme un personnage qui a voulu «oublier, anéantir quelque chose en lui, [...] son moi égoïste, sa petite personne» et s'est «rattaché à quelque chose de vaste auquel participait le fond de son être», ceci en vertu d'une vérité énoncée sous la forme d'un aphorisme: «Un homme a deux moi: l'un étroit, superficiel, qui l'isole du monde; l'autre souterrain, radical, qui le réunit au monde» ${ }^{32}$. En d'autres termes, l'égotisme peut mener à la force et à la plénitude de l'être, à condition de ne pas tomber dans le piège du romantisme, qui revient à utiliser sa sensibilité non pour transformer le monde, mais pour s'en détourner. Ainsi Boutros avoue sentir en lui «beaucoup de vanité et beaucoup d'égoïsme», tout en s'affirmant conscient «qu'on ne peut trouver de valeur à la vie que dans la mesure où l'on se renonce au profit d'une entreprise universelle», ce qui le conduit à conclure: «Peut-être un jour je mériterai d'être un chef, si je me suis d'abord dominé, brisé» ${ }^{33}$.

Drieu peint encore le mal et son remède, dans La Comédie de Charleroi. Dans la nouvelle qui donne son titre au recueil, le narrateur analyse lucidement son romantisme. Il se dépeint comme un «pauvre intellectuel confiné dans les bibliothèques», nouveau René qui rêve « de prolonger [s] es mois de vacances, [s] es mois de sauvagerie sur les grèves bretonnes» et se déclare accablé par une «mélancolie éternelle»; et comme de juste, dans les tranchées de la Grande Guerre, il se «rappell[e] avec transport la solitude de Fabrice à Waterloo» ${ }^{34}$. Mais il voit une issue à cette mélancolie moralement dissolvante: devenir «un homme à son plein; l'homme qui donne et qui prend dans la même éjaculation $»^{35}$, c'est-à-dire un chef. Devenir un chef, c'est pour

(29) Ibid., p. 91.

(30) Voir Rêveuse bourgeoisie [Gallimard, 1937], rééd. Gallimard, coll. «L'Imaginaire», 1995, p. 263, p. 267 et p. 236.

(31) Un Homme libre, in Romans et Voyages, t. I, Paris, Laffont, coll. «Bouquins», p. 102 et p. 148.

(32) Une Femme à sa fenêtre [Gallimard, 1929], rééd. Gallimard, coll. «Folio» n² 2835, 1996, p. 139.

(33) Ibid., p. 198.

(34) La Comédie de Charleroi [Gallimard, 1934], rééd. Gallimard, coll. «L'Imaginaire», 1996, p. 39 et p. 87 .

(35) Ibid., p. 63. 
Drieu réconcilier «l'action» et «la contemplation», comme il le montrera encore dans L'Homme à cheval, roman qui offre une méditation sur ces deux thèmes et esquisse cet idéal du chef -; c'est, en d'autres termes, retrouver l'équilibre entre le corps et l'esprit célébré dans Notes pour comprendre le siècle, ce qui suppose de tirer parti de la réaction anti-rationaliste qui est en germe dans le romantisme et s'épanouit - non sans se dégrader - dans le symbolisme.

D'une façon plus générale, c'est toute la création romanesque de Drieu, et en particulier les personnages qu'il invente, qui s'accordent à la vision du romantisme proposée dans Notes pour comprendre le siècle. D'un bout à l'autre de son ouvre, il met en scène des personnages à la fois épuisés, moralement plus que physiquement, et cependant capables de réagir dans un sursaut salutaire, en conciliant enfin action et contemplation. Ainsi de Boutros, le héros d'Une femme à sa fenêtre: obsédé par la décadence, comme Drieu, il rêve «des germinations de demain à travers les effondrements et les pourritures qui nous entraînent», cherche à tirer «une leçon spirituelle inconnue dans tous [1] es signes renouvelés que faisaient les atomes en folie» et parvient finalement, à Delphes, à «saisir les mouvements de l'énergie à travers le monde», en retrouvant «la spiritualité [qui] pour lui jaillissait de ces faits qui parlaient si bien à ses sens: la sueur, le sang, les cris, les odeurs des foules en marche derrière leurs états-majors hagards» ${ }^{36}$. De même que Boutros incarne exemplairement la possibilité de réagir contre la décadence en s'affranchissant du rationalisme et en tirant parti de cette mysticité qu'avait vu renaître le romantisme, de même Bagdad, dans Béloukia, peut apparaître comme le lieu emblématique de l'univers romanesque et de la vision du monde propres à Drieu, marqués à la fois par le sentiment de la décadence et la conviction d'un sursaut possible: «Bagdad était alors pleine comme toutes les villes civilisées qui commencent délicieusement à pourrir. Il y avait encore de l'énergie à Bagdad, mais déjà en se déformant elle produisait maints grotesques» ${ }^{37}$. L'extrême civilisation coïnciderait donc avec le triomphe de l'intelligence, de la culture, mais déterminerait fatalement une faiblesse morale: ce constat qui sous-tend le portrait des écrivains symbolistes dans Notes pour comprendre le siècle, voire l'analyse du XIX ${ }^{\mathrm{e}}$ siècle tout entier, n'est en réalité que la reprise d'un lieu commun propre au discours contre la décadence dans les années 1890.

Car c'est bien la conviction de la décadence, autrement dit, dans une certaine mesure: l'adhésion à ce que l'on peut considérer comme un mythe, un «fait d'imagination ${ }^{38}$ auto-alimenté par la littérature au tournant du siècle, qui façonne le jugement ambivalent porté par Drieu sur le romantisme. Le romantisme de sa jeunesse, comme celui de ses personnages, les jeunes gens de Rêveuse bourgeoisie, porte en lui les rêves d'un sursaut ou d'une réaction qui, du point de vue de Drieu, n'a pas eu lieu. D'une génération à l'autre, du romantisme au symbolisme, du symbolisme à la génération de Drieu et d'Aragon, nés à la fin des années 1890, il lui semble que l'homme gagne en intelligence, en culture et en spiritualité, autant de gages d'une réaction possible contre la décadence, mais qu'il échoue généralement à tirer profit de ce regain de spiritualité. L'évolution littéraire reflète pour lui le processus de décadence, qui pourtant ne se laisse entrevoir qu'au moment où l'on distingue la possibilité de s'y opposer. Mais elle témoigne surtout des progrès de ce processus, dans la mesure

(36) Une Femme à sa fenêtre, cit., p. 180, p. 178 et p. 242.

(37) Béloukia [Gallimard, 1936], rééd. Gallimard, coll. «L'Imaginaire», 1991, p. 37.

(38) Nous reprenons le constat de P. Ciтti, Contre la décadence. Histoire de l'imagination française dans le roman (1890-1914), Paris, Presses Univer- sitaires de France, 1987, p. 13: «Contentons-nous de voir un fait d'imagination dans le phénomène littéraire qui de Huysmans à Laforgue a fait prononcer le mot de "décadents". Soyons sûrs qu'il correspond à une face de la réalité, mais la réalité n'apparaît qu'à travers les représentations, dont elle représente la somme idéale». 
où le romantisme lui-même apparaît toujours plus dégradé au fil du temps en la personne de ses héritiers, parmi lesquels le romancier Drieu doit se compter, à son corps défendant: on conçoit dès lors que son œuvre, de son propre aveu, ait «connu un développement très difficile» ${ }^{39}$ et qu'elle soit marquée, de façon obsessionnelle, par les thèmes de l'échec et de l'impuissance.

JEAN-MICHEL WITTMANN

(39) Voir sa préface à Gilles, cit., p. 9. 